\title{
Potential existence of post-perovskite nitrides; DFT studies of $\mathrm{ThTaN}_{3}$
}

\author{
M. Samir S. F. ; M. Demazeau G.
}

Abtract : Within density functional theory, the equations of state for cubic perovskite (c-pv) and hypothetic orthorhombic perovskite (o-pv $\mathrm{GdFeO}_{3}$-type) and post-perovskite (ppv) forms of $\mathrm{ThTaN}_{3}$ are obtained. The decreasing volume and stabilizing energy indicate pressure enabled transitions: c-pv $\rightarrow 0-p v \rightarrow p p v$. From electronic structure analysis the chemical system is found insulating in the c-pv ground state form with $\approx 1 \mathrm{l} /$ band gap and semi-conducting for the ppv due to increased covalence. The chemical bonding properties show that Th and Ta bondings with the $2 \mathrm{~N}$ sites are selectively differentiated and reinforced for Ta-N bond within ppv form. This is the consequence of the corner as well as edge sharing octahedra characterizing ppv while pv structures have only corner sharing octahedra. It is the first case of potential post-perovskite nitride.

$\mathrm{ThTaN}_{3}$ : Projected charge density (for $4 \mathrm{fu}$ ) onto basal plane: (a) cubic perovskite, (b) orthorhombic perovskite and (c) post-perovskite. Red, green and blue areas are relevant to strong, medium and low localization of density.

\section{Introduction :}

The structural transformations of Minerals in deep Geosphere are an important challenge for explaining the structure and the properties of the Earth [1]. Taking into account that $\mathrm{MgSiO}_{3}$ and $(\mathrm{Mg}, \mathrm{Fe}) \mathrm{SiO}_{3}$ with the perovskite structure as the main components of the lower mantle [2] and due to the seismic anisotropy of the $D^{\prime \prime}$ layer at the boundary between the lower mantle and the outer core, the structural transformation of $\mathrm{MgSiO}_{3}$ was investigated by both Oganov and Ono [3] and Murakami et al. [4]. Both research works postulated the transformation of perovskite (pv) to the $\mathrm{CalrO}_{3}$-type structure, which was then called post-perovskite (ppv). The recent stabilizations of the ppv form for $\mathrm{CaRuO}_{3}$ by Kojitani et al. [5], $\mathrm{CaPtO}_{3}$ by Ohgushi et al. [6] and Inaguma et al. $\mathrm{CaRhO}_{3}$ by Shirako et al. [8] have extended the interest of the post-perovskite structure from Geosciences to Materials Chemistry. Through a simple analysis, two main factors seem to play an important role for stabilizing such a ppv structure for $\mathrm{ABO}_{3}$ :

- the degree of distortion of the perovskite-precursor (pv) with the $\mathrm{GdFeO}_{3}$-type (corresponding to the tilting of the $\mathrm{BO}_{6}$ octahedra and a small value for the Goldschmidt factor), and

- the covalence of the $\mathrm{B}-\mathrm{O}$ bond.

Consequently, the ppv structure can be observed for the $\mathrm{ABO}_{3}$ compositions involving a $d^{4}$ or a $d^{5} B$ element and an $A$ cation such as $\mathrm{Ca}^{2+}$. At the present time, due to the importance of oxides in the Earth mantle, mainly $\mathrm{ABO}_{3}$ compositions have been investigated. However, the $\mathrm{ppv}$ structure has been observed for $\mathrm{NaMgF}_{3}$ with the neighborite structure in a pressure range between 28 and $30 \mathrm{GPa}$ by Martin et al. [9]. Such a result opens new horizons for the interest of the ppv structure in materials chemistry. The pv structure (cubic, c-pv and orthorhombic, o-pv) is only characterized by shared corners octahedra and a three-dimensional (3D) network, while the ppv one, shown in Fig. 1, corresponds to a two-dimensional (2D) network where octahedra share both corners and edges. Consequently, compounds with ppv structure can be characterized by specific electronic properties. Recently, the metal-insulator transition observed in $\mathrm{Ca}_{1-\chi} \mathrm{Na}_{x} \mid \mathrm{IO}_{3}$ with the ppvstructure is an interesting example [10].

Considering the covalence of the $\mathrm{B}-\mathrm{O}$ bond as a critical factor for stabilizing the ppv structure, nitrides would appear as good candidates for developing novel ppv compounds. The number of nitrides with the perovskite structure is reduced, $\operatorname{ThTaN}_{3}$ being considered as the most known [11]. Therefore, it was important to validate through energy derived quantities from ab initio calculations whether pv-nitrides could adopt the ppv structure and in which conditions. This is herein investigated for the first time in archetype trinitride, $\mathrm{ThTaN}_{3}$ by considering firstly 
the actual cubic structure; such a structure could be metastable at room temperature conditions due to the high temperature required for preparing $\operatorname{ThTaN}_{3}\left(1400{ }^{\circ} \mathrm{C}\right)$ [11]. Then ab initio parameters for orthorhombic $\mathrm{GdFeO}_{3}-\mathrm{pv}$ and $\mathrm{CalrO}_{3}$-ppv are derived, following the factors given above. For this purpose we call for two complementary methods within the well established quantum mechanical framework of the density functional theory, DFT [12] and

\section{Computational methods :}

A pseudo-potential approach within the VASP code [14] and [15] is firstly used. Besides testing the calculations in their ability to yield the experimental parameters of $\mathrm{ThTaN}_{3}$ cubic perovskite [11], the method enables optimizing the structure and parameters for hypothetic orthorhombic pv and ppv phases, starting from known oxide systems. This follows from the structural geomimetism principle [16] with Th, Ta and $\mathrm{N}$ placed at $\mathrm{Ca}$, Rh and $\mathrm{O}$ sites, respectively, in $\mathrm{CaRhO}_{3}$ oxide [8]. Then the respective equations of states (EOS) are obtained to confront the equilibrium volumes and relative stabilities. This is done through energy-volume curves fitted with Birch EOS [17]. For these purposes, pseudo-potentials built within the PAW (projector augmented wave) method [18] and are used, due to the presence of thorium (5f-subshell). For the treatment of the effects of exchange and correlation a local density approximation (LDA) [20] has been adopted. Preliminary test calculations for c-pv with gradient functional GGA [21] potentials led to unfavorable total energy with respect to LDA calculations by $\sim 28 \mathrm{e} /$ per formula unit (fu). The calculations are converged at energy cut-offs of $500 \mathrm{eV}$ for cubic pv and $400 \mathrm{eV}$ for orthorhombic pv and ppv ThTaN ${ }_{3}$, respectively, for the plane-wave basis set. The k-point integration is carried out with a starting mesh of $4 \times 4 \times 4$ up to $8 \times 8 \times 8$ for best convergence and relaxation to zero strains. The Brillouin-zone integrals are approximated using a special $k$-point sampling following Blöchl [18].

For a full description of the electronic band structures and of chemical bonding, the scalar relativistic all-electrons augmented spherical wave (ASW) [22] and [23] method is used. Like in the calculations with pseudo-potentials, the exchange and correlation effects are accounted for with an LDA functional [24]. In the ASW method, the wave function is expanded in atom-centered augmented spherical waves, which are Hankel functions and numerical solutions of Schrödinger's equation, respectively, outside and inside the so-called augmentation spheres. In order to optimize the basis set, additional augmented spherical waves are placed at carefully selected interstitial sites (IS). The choice of these sites as well as the augmentation radii are automatically determined using the spheregeometry optimization algorithm [25]. Self-consistency is achieved by a highly efficient algorithm for convergence acceleration [26]. The Brillouin zone integrations are performed using the linear tetrahedron method with up to 1728 and 1469 k-points for cubic and orthorhombic phases, respectively [18] and [23]. In the minimal ASW basis set, we have chosen the outermost shells to represent the valence states using partial waves up to $I_{\text {max. }}+1=4$ for Th,

$I_{\text {max. }}+1=3$ for Ta and $I_{\text {max. }}+1=2$ for $N$ and IS. The completeness of the valence basis set is checked for charge convergence. The self-consistent field calculations are run to a convergence of $\Delta Q=10^{-8}$ for the charge density and the accuracy of the method is in the range of about $10^{-8} \mathrm{Ryd}(1 \mathrm{Ryd}=13.6 \mathrm{eV})$ regarding energy differences.

The relative magnitude of the chemical bonding is obtained based on the overlap population analysis: $S_{i j}, i$ and $j$ being two chemical species. The crystal orbital overlap population (COOP) [27] criterion is used as implemented within the ASW method [23]. For the purpose of establishing trends of chemical bonding strength, we show the integrated COOP (iCOOP): below the curves, a larger area indicates a larger bonding. In the plots positive, negative and zero iCOOP magnitudes indicate bonding, anti-bonding and non-bonding interactions, respectively.

\section{Equation of state and charge density}

\subsection{Geometry optimization and equation of state}

The results of full geometry relaxation for cubic and orthorhombic perovskites, resp. c-pv o-pv, and base centered orthorhombic ppv $\mathrm{ThTaN}_{3}$ are presented in Table 1. The optimized cell volume for c-pv shows a good agreement with experiment with a slightly smaller value. This can be expected from the use of the LDA exchange-correlation 
functional which tends to reduce cell spacings in so far it is based on the homogeneous electron gas distribution. The calculated volume per formula unit (fu), is $64.24 \AA^{3} / \mathrm{fu}$, i.e. $256.96 \AA^{3}$ for $4 \mathrm{fu}$, is larger than in o-pv. Regarding o-pv and ppv, the volume is calculated smaller for the latter as it is the case of oxide systems [5] and [8]. The volume decrease lets suggest that the transition: c-pv $\rightarrow 0-p v \rightarrow p p v$ could occur by the effect of pressure. In both o$\mathrm{pv}$ and ppv, the internal coordinates of Th (Ca), N1 (O1 and N2 (O2) are within range of those of o-pv and ppv oxide systems $\mathrm{CaRuO}_{3}$ [5] and $\mathrm{CaRhO}_{3}$ [8]. For the three forms, the corresponding energetics and derived quantities such as the zero pressure bulk modulus $B_{0}$, need to be examined further for establishing trends.

Table 1.

$\mathrm{ThTaN}_{3}$ : experimental data for c-pv are from Ref. [11].

\begin{tabular}{|c|c|c|c|}
\hline $\mathrm{ThTaN}_{3}$ & c-pv- $-P m 3 n$ & o-pv-Pnma & ppv-Cmcm \\
\hline a-latt. const. $(\AA)$ & $4.02 / 4.01$ & $-/ 5.55$ & $-/ 3.12$ \\
\hline b-latt. const. ( $\AA$ ) & - & -15.65 & $-/ 10.15$ \\
\hline c-latt. const. (Å) & - & $-/ 7.89$ & $-/ 7.44$ \\
\hline Volume $\left(\AA^{3}\right)$ & $64.96 / 64.24$ & $-/ 247.41$ & $-/ 235.61$ \\
\hline \multicolumn{4}{|l|}{ Atoms } \\
\hline Th & $0,0,0$ & $0.044, \frac{1}{4}, 0.994$ & $0,0.26, \frac{1}{4}$ \\
\hline Ta & $\frac{1}{2}=\frac{1}{2}=\frac{1}{2}$ & $0,0, \frac{1}{2}$ & $0,0,0$ \\
\hline N1 & $\frac{1}{2}, \frac{1}{2}, 0$ & $0.48, \frac{1}{4}, 0.09$ & $\frac{1}{2}, 0.43, \frac{1}{4}$ \\
\hline $\mathrm{N} 2$ & $0, \frac{1}{2}=\frac{1}{2} ; \frac{1}{2}, 0 \frac{1}{2}$ & $0.30,0.047,0.70$ & $\frac{1}{2}, 0.131,0.057$ \\
\hline \multicolumn{4}{|l|}{ Distances $(\AA)$} \\
\hline Th...N1 & $3.48 / 3.46$ & $-/ 2.34 / 2.50$ & -12.33 \\
\hline Th....N2 & & $-/ 2.38 / 2.71 / 2.75$ & $-/ 2.49$ \\
\hline Ta...N1 & $2.01 / 2.00$ & $-/ 2.04$ & -12.00 \\
\hline Ta...N2 & & $-/ 2.06$ & $-/ 2.10$ \\
\hline
\end{tabular}

Starting coordinates for o-pv and ppv are from [5] and [8]. Calculated values are in italics. Note that N1 and N2 are equivalent in cubic perovskite, pv-Pmßn .

Let us mention here that the calculated total energy pertains to the cohesive energy within the crystal because the solution of the Kohn-Sham DFT equations yields the energy with respect to infinitely separated electrons and nuclei. In as far as the zero of energy depends on the choice of the pseudo-potentials, somehow it becomes arbitrary; i.e. it is shifted, not scaled. However, the energy derivatives remain unaltered as well as the equations of state. For this reason one needs to establish the EOS and extract the fit parameters for an assessment of the equilibrium values. This can be done from a set of $E(V)$ calculations for all three forms. The resulting curves shown in Fig. 2 have a quadratic variation which can be fitted with Birch EOS to the 3rd order [17]: 


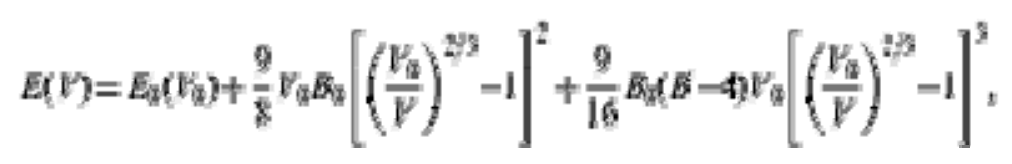

where $E_{0}, V_{0}, B_{0}$ and $\underline{B}^{\prime}$ are the equilibrium energy, the volume, the bulk modulus and its pressure derivative, respectively. The Birch EOS is normally employed by assuming the following trend: the larger the value of $B_{0}$, the harder is the material. The fit results given in the insert of Fig. 2 for 1 formula unit (fu), indicate the same trend of volume decrease c-pv $\rightarrow 0-p v \rightarrow p p v$ although not with the same magnitudes for the reasons stated above. Further information is obtained regarding the energetics, whereby the equilibrium energy decreases with decreasing volume from c-pv $(-55.44 \mathrm{eV})$ to o-pv $(-55.68 \mathrm{eV})$ and ppv $(-55.79 \mathrm{eV})$. Consequently, the largest amount of stabilization is due to the distortion c-pv $\rightarrow$ o-pv, i.e., with the orthorhombicity inducing the tilting and distortion of the octahedra. In a subsequent step, the arrangement of the octahedra into edge sharing ones from o-pv $\rightarrow \mathrm{ppv}$ is induced with less energy: $\sim-0,11 \mathrm{eV} / \mathrm{f}$ : While the distortion c-pv $\rightarrow 0-p v$ is not envisaged in $\mathrm{CaRuO}_{3}[5]$ and $\mathrm{CaRhO}_{3}[8]$, the energy involved in the o-pv $\rightarrow$ ppv transformation in these oxides is higher $\left(\sim-0 . \mathrm{fieV}_{/} / \mathrm{fin}\right)$ with respect to the studied nitride system. This could be due to the already high covalence of the metal-nitrogen chemical bond with respect to metal-oxygen one. Also the small energy differences makes the curves cross each other in Fig. 2 with two main volume regions, at high volume the c-pv phase is stable and at high volume the ppv is stabilized. This indicates that the transformation $\mathrm{pv} \rightarrow \mathrm{ppv}$ needs to be helped with pressure. The volume decrease following the sequence $\mathrm{c}-\mathrm{pv} \rightarrow \mathrm{o}-\mathrm{pv} \rightarrow \mathrm{ppv}$, is concomitant with an increasingly larger $\mathrm{B}_{o}$ bulk modules: c-pv (260 GPa), o-pv (262 GPa) and ppv (268 GPa). These values are within range albeit with slightly larger magnitudes, of oxide pv and ppv systems [28] and [29]. In all forms, the magnitude of $B^{\prime}$ amounts to $\sim \underline{4}$, a value usually encountered [30].

The transformation c-pv $\rightarrow$ o-pv with pressure can be further addressed based on the relative compressibilities of the $A X_{12}$ and $B X_{6}$ polyhedra (here $A=\mathrm{Th}, B=\mathrm{Ta}$ and $X=\mathrm{N}$ ) [31] and If $A X_{12}$ polyhedron is more compressible than $B X_{6}$ one, the orthorhombic distortion occurs; if the trend is opposite the cubic form should stabilize. From Table 1 the larger change of Th-N separation versus Ta-N from c-pv to o-pv would support the first hypothesis of the orthorhombic distortion as the derived energetics let suggest.

\subsection{Charge density projections}

We can illustrate the results with a plot of the charge density resulting from the calculations. This is shown for a projection onto basal planes for all three forms in Fig. 3 considering 4 fu for sake of clarity. Blue, green and red areas indicate low, medium and strong charge densities, respectively. For a guide of the eye the projections show the Ta- and $\mathrm{N}$-centered densities. While regular octahedra projections can be traced out in c-pv (Fig. 2a), these are tilted in o-pv (Fig. 3b). But the major difference appears for ppv (Fig. 3c), where the octahedra are now edge sharing. Note that the ppv $\mathrm{C}$ symmetry space group allows to show a non-conventional projection of a losange-like projection. From Fig. 3c, the density spots are blue-green within $\mathrm{Ta}_{2} \mathrm{~N}_{2}$-like motifs, i.e. with medium density, while very low density regions (blue) are observed between the layers due to the 2D-like arrangement of octahedra in ppv. This is also stressed in the complementary projection of Fig. 3c showing a chain of ... $\mathrm{N}-\mathrm{N}-\mathrm{Ta}-\mathrm{N}-\mathrm{N}-\mathrm{Ta}$... array as in Fig. 1. This illustrates furthermore the discussion of the difference between pv and ppv presented in the introduction. Then, one may expect enhanced covalence within the latter (cf. chemical bonding section). Lastly, despite the layer-like ppv structure, its slightly larger $B_{0}$ value could be due to the improved compactness of the octahedra within the layer in as far as they are assembled into corner as well as edge sharing motifs. In other words the system should present a larger incompressibility within the layers than between them, so that the average incompressibility is likely to be higher. 


\section{Electronic structure and chemical bonding}

Using the geometry optimized lattice parameters of Table 1 for c-pv, o-pv and ppv, we have computed in a second step the electronic structures as well as the properties of chemical bonding with the scalar relativistic all-electrons augmented spherical wave (ASW) method [22] and [23]. At self-consistent convergence, charge transfer is observed from Th and Ta toward nitrogen and IS with amounts of $1-2$ electrons. These transfers are not meaningful of an ionic character such as $\mathrm{N}^{3-}$ as one would expect from the formal ionic $\mathrm{Th}^{4+} \mathrm{Ta}^{5+}\left(\mathrm{N}^{3-}\right)_{3}$ chemical picture, not encountered in such calculations. Further this points to a covalently bonded nitride system. It will be shown that in such a chemical system the physical properties are obtained from a contribution of all present species due to the quantum mixing (hybridization) between the valence states.

\subsection{Density of states}

The site projected density of states (PDOS) are shown at Fig. 4. In these plots and in following ones the energy reference along the $x$ axis is with respect to the top of the valence band (VB), $E_{V}$, in as far as the chemical system is computed to be insulating with a gap of $\sim 1 \mathrm{eV}$. The presence of a gap results from the absence of hole in the VB, just like in $\mathrm{CaPtO}_{3}$ calculated within same framework in o-pv and ppv structures [28]. However, the band gaps in the oxide are much larger than in the nitride for covalence criteria. The multiplicities of all atomic constituents are accounted for in the plots, ex. the DOS intensity is higher in o-pv due to a twice larger unit cell with respect to ppv. Early actinide Th has its $f$ subshell centered within the conduction band but there are itinerant delocalized states (as opposed to localized states such $5 f$ ) which extend the thorium PDOS below $E_{V}$, within VB, mainly in ppv. The valence band is dominated by metal (Th, Ta) and N PDOS. They mix within low lying $s, p N$-like states around $-15 \mathrm{eV}$ and $\mathrm{d}$ Ta-like in the energy window $\left[-5 \mathrm{eV}, E_{V}\right]$. Interestingly, the width of the $s, p$ band increases from $\sim 3 \mathrm{eV}$ in Fig. $4 \mathrm{a}$, to $\sim 4 \mathrm{eV}$ in Fig. $4 \mathrm{~b}$ and $\sim 5 \mathrm{eV}$ in Fig. 4c. This is an indication of the increased covalence in the series: c-pv $\rightarrow 0-p v \rightarrow p p v$. Such a feature is also observed within the CB where itinerant states such as s-like ones tend to reduce the energy gap leading to a semi-conducting behavior.

\subsection{Chemical bonding}

The DOS results can be further assessed in a chemical bonding qualitative analysis, focusing on the bonding between 'cationic' Th and Ta on one hand and 'anionic' N (N1 and N2, cf. Table 1) on the other hand. In order to establish qualitative comparisons of bonding strengths: the larger the iCOOP area below the curves the larger the bonding. One atom of each species is considered regardless of its multiplicity in the respective SG's. The plots are shown in Fig. 5. The upper panel of cubic perovskite which has only one $\mathrm{N}$ sublattice, shows the expected feature of a stronger Ta-N than Th-N bond with a larger area underneath Ta-N curve. This is due to distance criteria, i.e.

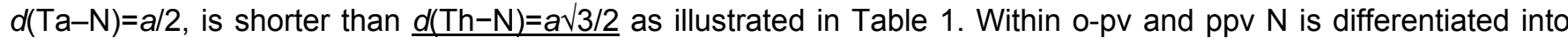
two sublattices N1 and N2 (cf. Table 1). Then the interactions are 4-fold resulting in four curves in Fig. 5b and c, respectively. In o-pv iCOOP plots, the general trend is towarda stronger overall Ta-N bonding versus Th- $\mathrm{N}$ one. This also follows from distance criteria whereby the average distance $d(\mathrm{Ta}-\mathrm{N})=2.05 \AA$ is smaller than $d(\mathrm{Th}-$ $\mathrm{N})=2.36 \AA$. However, the larger $i \mathrm{COOP}$ for Th and Ta with N2 sublattice is a feature that cannot be related with distance considerations; the other mechanism that can be invoked is chemical, whereby the covalence is increased for the bonding with N2 sublattice. This is largely enhanced in ppv iCOOP (Fig. 4c) with the strongest bonding observed for Ta-N2 (notice the twice larger scale of $y$-axis) despite the close magnitudes of Ta-N1 and Ta-N2 distances. Further, this enhancement is much larger than in ppv oxides as $\mathrm{CaPtO}_{3}$ [28] and $\mathrm{CaRhO}_{3}$ [29]. Then the anisotropy of the chemical bonding is stronger within $M N_{6}$ versus $M O_{6}$ ones. This reinforcement of the Ta-N2 bond should induce interesting physical properties. The Th-N1/Th-N2 bonding is as in o-pv meaning that the o-pv and ppv transformation affects less the Th- $\mathrm{N}$ interactions than Ta-N ones. We suggest this feature to be resulting from the edge sharing octahedra in ppv. 


\section{Conclusion}

Using theoretical modeling tools within DFT, the equations of state (EOS) of perovskite (c-pv and o-pv) and postperovskite (ppv) $\mathrm{ThTaN}_{3}$ have been derived. The energetics and volume trends let suggest a pressure enabled transition in the sequence: $c-p v \rightarrow 0-p v \rightarrow p p v$. The required transition c-pv $\rightarrow 0-p v$ before the stabilization of the $p p v$ form could be due to the metastability of the c-pv form at room temperature. An examination of the electronic and chemical bonding properties shows an increased covalence within ppv due to corner as well as edge sharing $\operatorname{TaN}_{6}$ octahedra contrary to c- and o-pv which show only corner sharing octahedra. This is made explicit for the different $\mathrm{Ta}$ and Th interactions with the two $\mathrm{N}$ sublattices from a qualitative analysis of bonding strengths. Due to covalence of the $\mathrm{M}-\mathrm{N}$ bonds compared to $\mathrm{M}-\mathrm{O}$ ones, the possible stabilization of nitrides with the ppv structure opens new horizons for materials. Besides computations, it can be proposed that the phase transformation is likely triggered by the covalence of the nitride, increasingly large for the $\mathrm{Ta}-\mathrm{N}$ sublattice on one hand and larger than in oxide homologs on the other hand.

\section{Acknowledgments}

Computational facilities were provided by "Mésocentre de Calcul Intensif Aquitain" (MCIA)—University of Bordeaux (http://www.m3pec.u-bordeaux1.fr), financed by the "Conseil Régional d'Aquitaine", the "Ministère de la Recherche et de l'Enseignement Supérieur" and the "Institut des Grilles du CNRS".

\section{References}

[1] D.Yu. Pushcharovsky, R. Oganov. 51 (2006) 767.

[2] G. Fiquet, Z. Krist. 216 (2001) 248.

[3] A.R. Oganov and S. Ono, Nature 430 (2004), p. 445.

[4] M. Murakami, K. Hirose, K. Kawamura, N. Sata and Y. Ohishi, Science 304 (2004), p. 855.

[5] H. Kojitani, Y. Shirako and M. Akaogi, Phys. Earth Planet. Interiors 165 (2007), p. 127.

[6] K. Ohgushi, Y. Matsushita, N. Miyajima, Y. Katsuya, M. Tanaka, F. Izumi, H. Gotou, Y. Ueda and T. Yagi, Phys. Chem. Min. 35 (2008), p. 189.

[7] Y. Inaguma, K-I. Hasumi, M. Yishida, T. Ohba and T. Katsumata, Inorg. Chem. 47 (2008), p. 1868.

[8] Y. Shirako, H. Kojitani, M. Akaogi, K. Yamaura and E. Takayama-Muromachi, Phys. Chem. Min. 36 (2009), p. 455.

[9] C.D. Martin, Y. Meng, V. Prakapenka and J.B. Parise, J. Appl. Crystallogra. 41 (2008), p. 38.

[10] K. Ohgushi, H. Gotou, T. Yagi, Y. Kiuchi, F. Sakai and Y. Ueda, Phys. Rev. B 74 (2006), p. 241104.

[11] N.E. Brese and F.J. DiSalvo, J. Solid State Chem. 128 (1995), p. 378.

[12] P. Hohenberg and W. Kohn, Phys. Rev. B 136 (1964), p. 864.

[13] W. Kohn and L.J. Sham, Phys. Rev. A 140 (1965), p. 1133.

[14] G. Kresse and J. Furthmüller, Phys. Rev. B 54 (1996), p. 11169.

[15] G. Kresse and J. Joubert, Phys. Rev. B 59 (1999), p. 1758.

[16] S.F. Matar, E. Betranhandy, M. Nakhl, M. Zakhour-Nakhl and N. Ouaïni, Prog. Solid State Chem. 34 (2006), p. 21.

[17] F. Birch, J. Geophys. Res. 83 (1978), p. 1257.

[18] P.E. Blöchl, Phys. Rev. B 50 (1994), p. 17953.

[19] G. Kresse and J. Joubert, Phys. Rev. B 59 (1999), p. 1758.

[20] D.M. Ceperley and B.J. Alder, Phys. Rev. Lett. 45 (1980), p. 566.

[21] J.P. Perdew, S. Bürke and M. Ernzerhof, Phys. Rev. Lett. 77 (1996), p. 3865.

[22] A.R. Williams, J. Kübler and C.D. Gelatt, Phys. Rev. B 19 (1979), p. 6094.

[23] V. Eyert, The Augmented Spherical Wave Method-A Comprehensive Treatment, Lecture Notes in Physics vol. 719, Springer, Berlin, Heidelberg (2007).

[24] S.H. Vosko and L. Wilk, M. Nusair. Can. J. Phys. 58 (1980), p. 1200.

[25] V. Eyert and K.-H. Höck, Phys. Rev. B 57 (1998), p. 12727.

[26] V. Eyert, J. Comput. Phys. 124 (1996), p. 271.

[27] R. Hoffmann, Angew. Chem. Int. Ed. Engl. 26 (1987), p. 846.

[28] S.F. Matar, G. Demazeau and A. Largeteau, Chem. Phys. 352 (2008), p. 92. 
[29] S.F. Matar, G. Demazeau and A. Largeteau, Solid State Sci. 12 (2010), p. 373.

[30] S. Stølen and R. Tronnes, Phys. Earth Planet Interiors 164 (2007), p. 50.

[31] E. Keller and V. Krämer, Acta Cryst. B 62 (2006), p. 411.

[32] J. Zhao, N.L. Ross and R.J. Angel, Acta Cryst. B 62 (2006), p. 431.

This paper is dedicated to Engineer Simon T. Khoury, inventor, for all his works. 


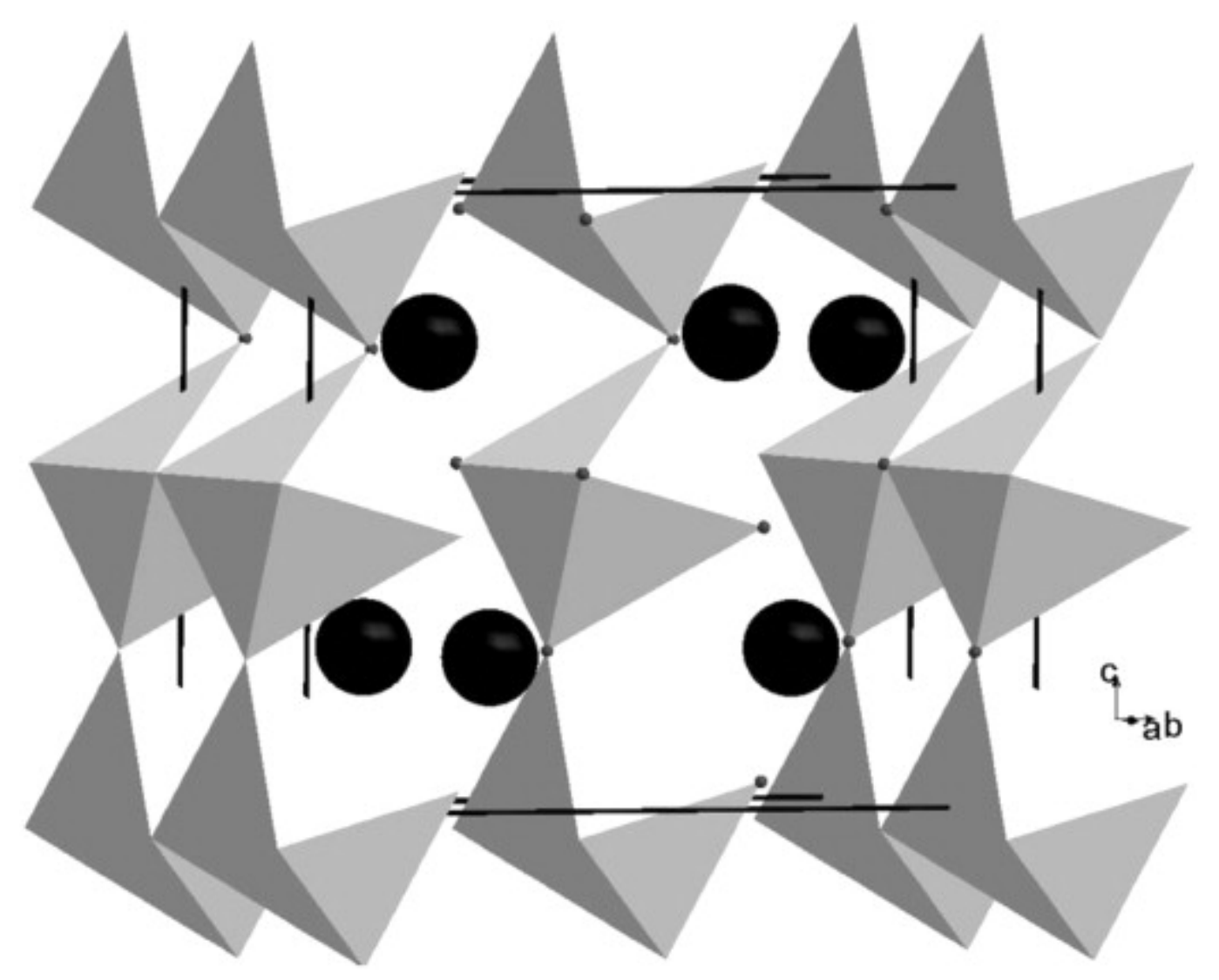

Figure1. : Arrangement of edge sharing tilted $\mathrm{BX}_{6}$ octahedra in the $\mathrm{Cmcm}$ orthorhombic post perovskite structure $A B X_{3}$ with $B=T a$ and $X=N$ here. Black spheres between the octahedra represent large $A(T h)$ cation. Lattice vectors $\mathrm{a}, \mathrm{b}, \mathrm{c}$ correspond with data in Table 1.

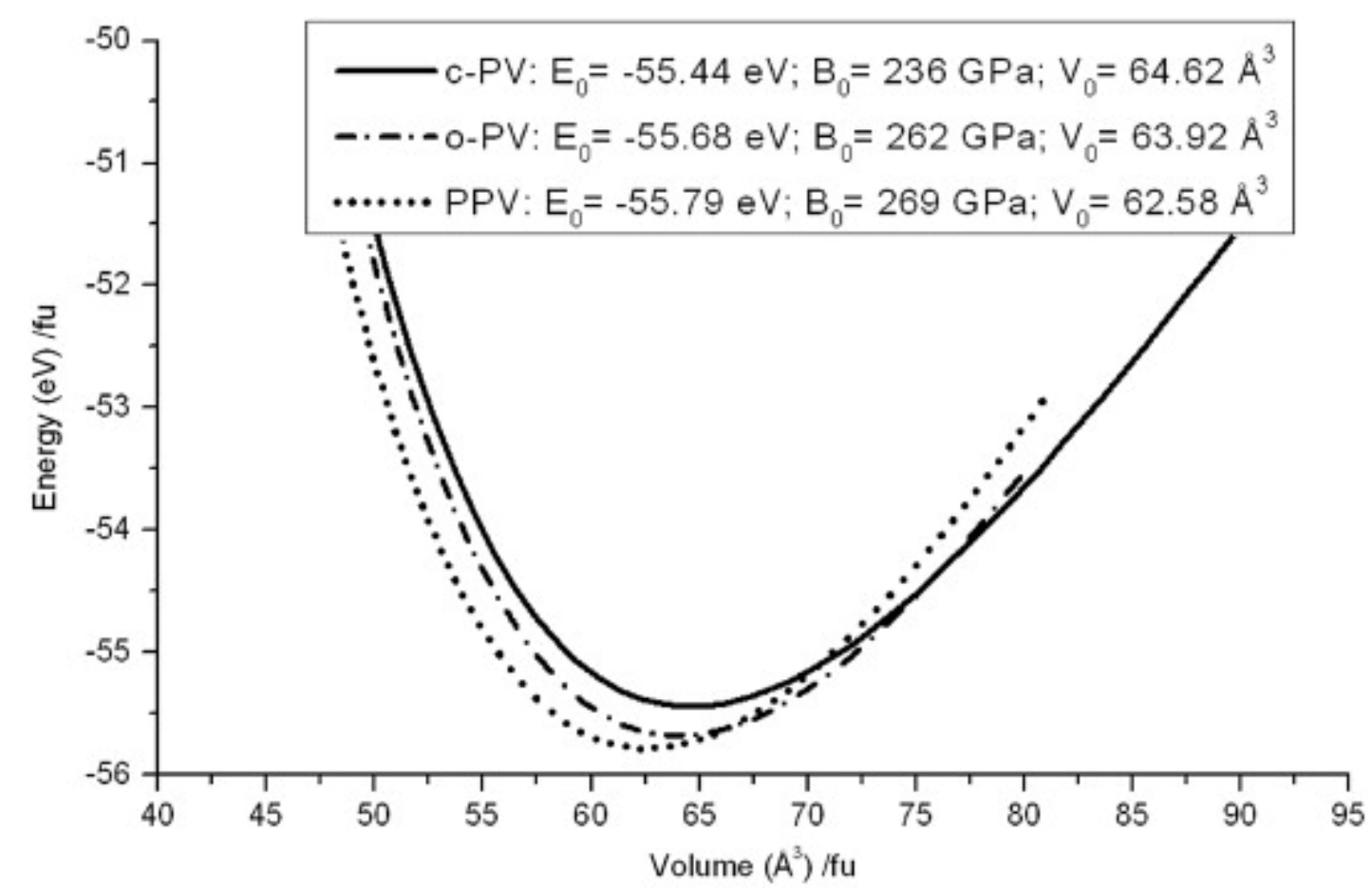

Figure $2: \mathrm{ThTaN}_{3}$ : total energy versus cell volume curves, for c-pv, o-pv and ppv. Fit values are per formula unit (fu) from Birch 3rd order EOS. 

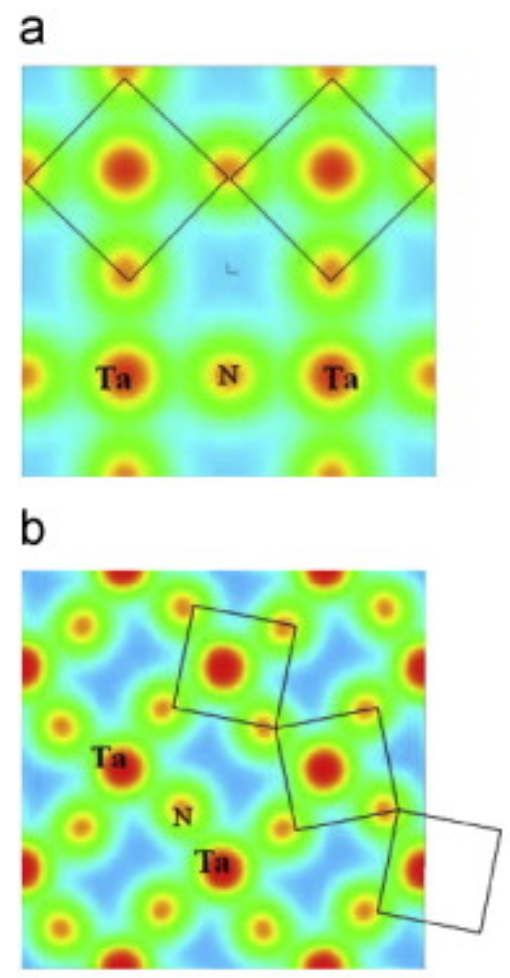

C

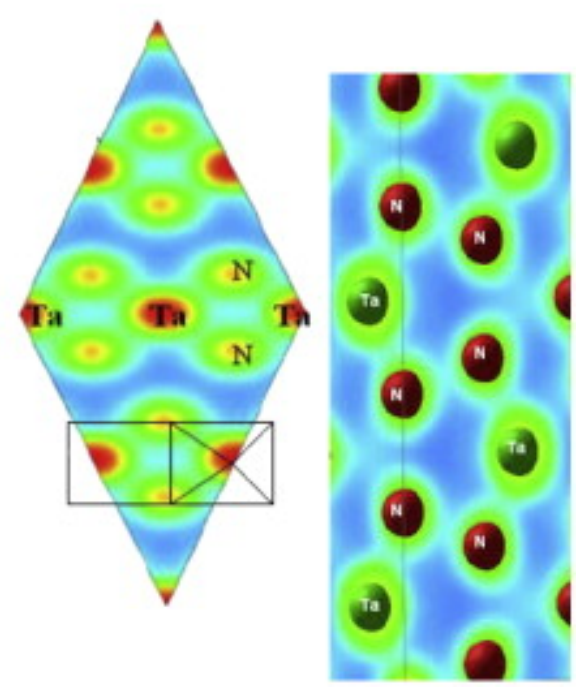

Figure 3 : ThTaN $_{3}$ : projected charge density (for $4 \mathrm{fu}$ ) onto basal plane of c-pv (a), o-pv (b) and ppv (c). Red, green and blue areas are relevant to strong, medium and low densities. Notice the twisting of the corner sharing TaN $\mathrm{N}_{6}$ like octahedra (projected as squares) from (a) to (b) and the edge sharing octahedra in (c); also shown on the right hand panel the projection of chains of tilted octahedra with ...Ta-N-N-Ta-N-N-Ta... sequence as in Fig. 1. See text for details. (For interpretation of the references to color in this figure legend, the reader is referred to the web version of this article.) 

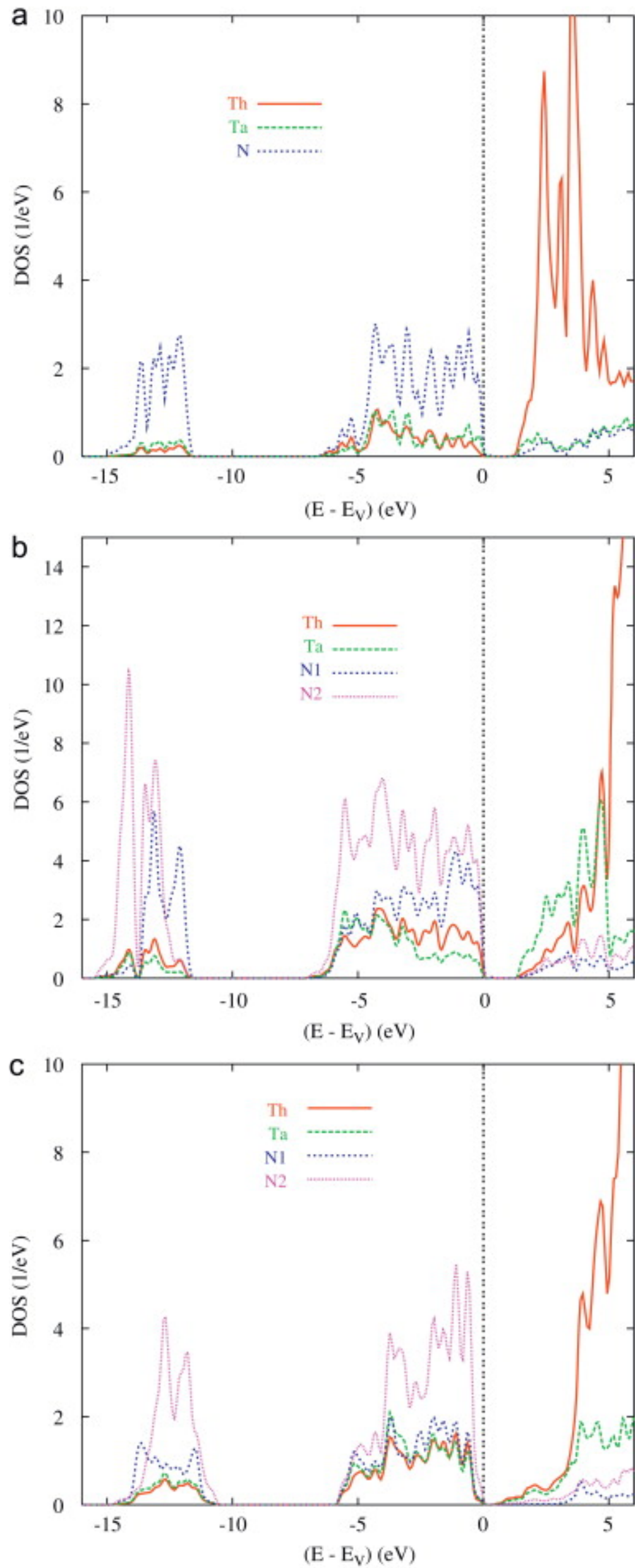

Figure 4 : (Color online) $\operatorname{ThTaN}_{3}$ : site projected density of states for c-pv (a), o-pv (b) and ppv (c). Zero energy is with respect to the top of the valence band. Site multiplicities of atoms in respective structures are accounted for. 

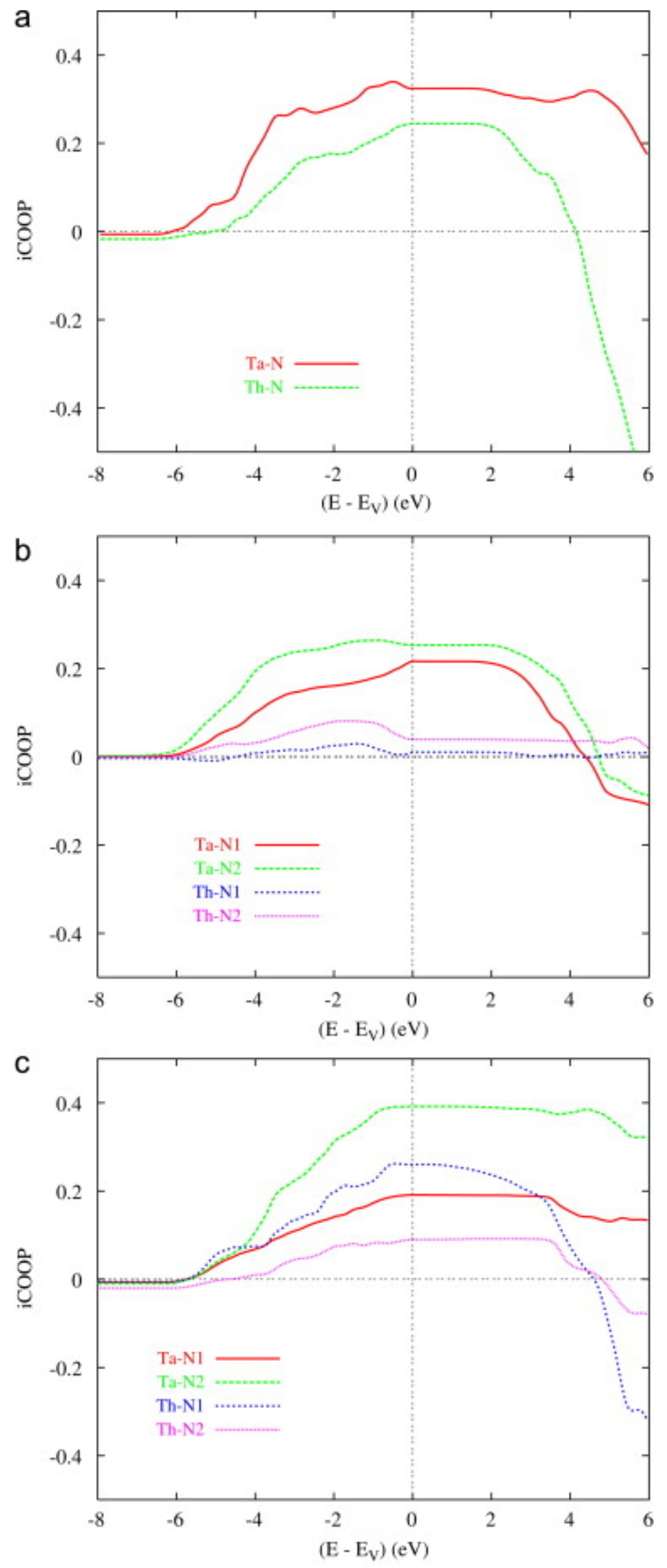

Figure 5 : (Color online) Integrated COOP (unit less, along $y$-axis) for metal-nitrogen bonding in $\mathrm{ThTaN}_{3}$ : (a) c-pv, (b) o-pv and (c) ppv. Along $x$-axis zero energy is with respect to the top of the valence band. 